\title{
Exocytosis of Vesicular Zinc Reveals Persistent Depression of Neurotransmitter Release during Metabotropic Glutamate Receptor Long-Term Depression at the Hippocampal CA3-CA1 Synapse
}

\author{
Jing Qian and Jeffrey L. Noebels \\ Department of Neurology, Baylor College of Medicine, Houston, Texas 77030
}

\begin{abstract}
Exocytosis can be directly measured in mammalian brain slices by fluorescence detection of vesicular zinc release. Detection of the low-level evoked zinc signal $[\mathrm{Zn}]_{\mathrm{t}}$ was first demonstrated at the zinc-rich hippocampal mossy fiber pathway and required the use of high-frequency presynaptic stimulation. Here, we show that release after individual action potentials can be reliably detected even at non-mossy fiber, zinc-poor synapses in the hippocampus, a major enhancement in the temporal resolution of the technique. Short-term facilitation of release properties of zinc-positive CA3-CA1 Schaffer collateral/commissural synapses in the stratum radiatum differ from those at mossy fibers but are similar to those measured for the EPSP [field EPSP (fEPSP)]. The N-type $\mathrm{Ca}^{2+}$ channel toxin $\omega$-conotoxin GVIA inhibited both the $[\mathrm{Zn}]_{\mathrm{t}}$ and fEPSP equally, and the modulation of neurotransmitter release by neuropeptide $\mathrm{Y}$, baclofen, and adenosine as revealed by $[\mathrm{Zn}]_{t}$ closely resembles that measured for the fEPSP. A long-standing controversy in hippocampal synaptic plasticity involves the site of long-term depression (LTD) at these synapses. Using zinc release as a direct marker for exocytotic events and a surrogate marker for glutamate release, we demonstrate that persistent depression of presynaptic release occurs in the late expression of DHPG [(S)-3,5-dihydroxyphenylglycine]-induced LTD at this synapse. The ability to examine release dynamics with zinc fluorescence detection will facilitate exploration of the molecular pharmacology and plasticity of exocytosis at many CNS synapses.
\end{abstract}

Key words: vesicular zinc release; FluoZin-3; plasticity; LTD; DHPG; hippocampus

\section{Introduction}

Metabotropic glutamate receptors (mGluRs) play a critical role in synaptic plasticity in the CNS (Fagni et al., 2000). In the hippocampal Schaffer collateral/commissural pathway, long-lasting depression of synaptic transmission [long-term depression (LTD)] can be induced by activating mGluRs at a low frequency with synaptically released glutamate (Bolshakov and Siegelbaum, 1994; Oliet et al., 1997) or with exogenously applied (S)-3,5dihydroxyphenylglycine (DHPG), an agonist of mGluRs. Although the induction of mGluR-LTD is widely thought to be postsynaptic, a definitive site for LTD expression has not been confirmed. Evidence for internalization or redistribution of ionotropic glutamate receptors and a requirement for postsynaptic protein synthesis in cultured neurons after application of DHPG supports a postsynaptic mechanism underlying mGluRLTD (Snyder et al., 2001; Xiao et al., 2001); however, more recent evidence, including altered paired-pulse facilitation (PPF), decreased miniature EPSC (mEPSC) frequency, and the absence of

Received Feb. 1, 2006; revised April 28, 2006; accepted May 2, 2006

This work was supported by National Institutes of Health-National Institute of Neurological Disorders and Stroke Grant NS29709.

Correspondence should be addressed to Dr. Jeffrey L. Noebels, Department of Neurology, Baylor College of Medicine, One Baylor Plaza, Houston, TX 77030. E-mail: jnoebels@bcm.tmc.edu.

D01:10.1523/JNEUROSCI.0475-06.2006

Copyright $\odot 2006$ Society for Neuroscience $\quad$ 0270-6474/06/266089-07\$15.00/0 a change in postsynaptic AMPA receptor sensitivity by DHPG all favor presynaptic expression of DHPG-LTD (Fitzjohn et al., 2001; Faas et al., 2002; Rammes et al., 2003; Rouach and Nicoll, 2003; Nosyreva and Huber, 2005). Recently, a developmental switch in the mechanism of mGluR-LTD expression has been proposed, based on evidence that mGluR-LTD at neonatal synapses is mediated by presynaptic mechanisms, whereas mGluRLTD at mature synapses may result from postsynaptic modifications (Nosyreva and Huber, 2005). Although DHPG is widely used to study mGluR-LTD and DHPG-LTD shares many features with synaptically induced mGluR-LTD, there are some unusual properties of DHPG-LTD. The DHPG-LTD expression requires sustained activation of mGluR (Watabe et al., 2002; Rouach and Nicoll, 2003; Huang and Hsu, 2006) and the induction of DHPG-LTD is independent of postsynaptic $\mathrm{Ca}^{2+}$ entry (Fitzjohn et al., 2001).

The ability to directly examine presynaptic neurotransmitter release rather than inferring changes in evoked quantal output by analysis of postsynaptic responses can provide critical information in localizing the site of plasticity at synapses. We recently introduced such a method based on fluorescence detection of vesicular zinc by an extracellular zinc indicator dye (Qian and Noebels, 2005). Zinc has been shown to coexist with glutamate within synaptic vesicles in glutamatergic synapses in the many parts of brain including the hippocampus (Frederickson et al., 
2000). Fluorescence detection of exocytosis by monitoring evoked vesicular zinc signals provides a novel way to assess neurotransmitter release at the zinc-containing hippocampal mossy fiber synapse (Qian and Noebels, 2005).

In the present study, we adapted the techniques developed for the mossy fiber synapse to measure vesicular zinc release evoked by individual action potentials at CA3-CA1 synapses within the hippocampal stratum radiatum. Zinc histochemistry detects a low level of vesicular zinc-containing terminals within this region. Recent analysis reveals that these synapses can be divided into two populations, those containing vesicular zinc and those without (Sindreu et al., 2003). The release properties of these populations have never been selectively characterized. We now demonstrate that fluorescence detection of zinc release can selectively resolve neurotransmitter release dynamics in the Schaffer collateral/commissural pathway, and apply this direct measure to examine DHPG-LTD at CA3-CA1 synapses. Our experimental results demonstrate a persistent depression of neurotransmitter release during DHPG-LTD at the zinc-containing mouse hippocampal CA3-CA1 synapse.

\section{Materials and Methods}

Preparation of brain slices. All procedures were performed in accordance with the guidelines of the National Institutes of Health, as approved by the Animal Care and Use Committee of Baylor College of Medicine. Transverse brain slices [200-250 $\mu \mathrm{m}$ thickness: $200 \mu \mathrm{m}$, optical recording; $250 \mu \mathrm{m}$, extracellular recording of field EPSP (fEPSP)] were prepared from hippocampi of wild-type (C57BL/6J) and Znt3-/- (C57BL/ $6 \times 129 / \mathrm{SvEv})$ mice. For the adult mouse group, the animal age ranges between 6 and 10 weeks. For the young mouse group in the LTD experiments, the animal age is between 15 and $18 \mathrm{~d}$. Znt3-/- mutant mice were generously provided by R. D. Palmiter (University of Washington, Seattle, WA). Brain slices were incubated in artificial CSF (ACSF) at $32^{\circ} \mathrm{C}$ for $1 \mathrm{~h}$ and then transferred into a submerged recording chamber mounted on an inverted microscope (Axiovert 100; Zeiss, Oberkochen, Germany). The ACSF contained the following (in $\mathrm{mm}$ ): $119 \mathrm{NaCl}, 2.5$ $\mathrm{KCl}, 2.5 \mathrm{CaCl}_{2}, 26 \mathrm{NaHCO}_{3}, 2 \mathrm{MgSO}_{4}, 1 \mathrm{NaH}_{2} \mathrm{PO}_{4}, 11$ D-glucose, 0.2 Ca-EDTA gassed with $95 \% \mathrm{O}_{2} / 5 \% \mathrm{CO}_{2}$ to maintain a constant $\mathrm{pH}$ of 7.4. The temperature of the recording chamber was controlled at $30^{\circ} \mathrm{C}$.

Zinc fluorescence detection in stratum radiatum of the CA1 region. A bipolar tungsten electrode was positioned in the stratum radiatum to stimulate the CA3-CA1 synapse. The stimulation pulse intensity was adjusted to $20 \mu \mathrm{A} / 0.1 \mathrm{~ms}$ to evoke a submaximal response for optical recording and to $10 \mu \mathrm{A} / 0.1 \mathrm{~ms}$ for extracellular recording of the fEPSP. A train of five pulses at $5 \mathrm{~Hz}$ was used to evoke the transient zinc fluorescence signal $\left(\Delta F\right.$ or $\left.[\mathrm{Zn}]_{\mathrm{t}}\right)$ and fEPSP in most experiments. The zincsensitive indicator FluoZin-3 was present in the extracellular solution at a concentration of $2 \mu \mathrm{M}$ for fluorescence recordings. The slow zinc chelator Ca-EDTA $(0.2 \mathrm{~mm})$ was routinely included in all experiments to reduce basal zinc fluorescence. The glutamate receptor antagonists CNQX and D-APV were also present in the perfusate during zinc measurement to eliminate the interference of autofluorescence. A small recording area ( $150 \mu \mathrm{m}$ in diameter) in the stratum radiatum of the CA1 region located $\sim 400 \mu \mathrm{m}$ away from the stimulation electrode was excited at a wavelength of $488 / 20 \mathrm{~nm}$; the emitted fluorescence was filtered by a bandpass filter of 535/25 nm and converted into electrical signals with a single photodiode. Because the zinc signal was obtained after abolishing the fEPSP, a separate set of slices was used to monitor glutamate release by field recording. Sharp glass recording electrodes (filled with $2 \mathrm{M} \mathrm{NaCl}$ ) were positioned at the same distance from the stimulation electrode as for optical recording. Signals were filtered through low-pass filters with a corner frequency of $5 \mathrm{kHz}$ and sampled at $10 \mathrm{kHz}$. Sample traces shown are an average of 5-10 successive traces during steady state to improve the signal-to-noise ratio. The initial slope of the fEPSP is taken as the measure of synaptic transmission. Because of a possible accumulation of extracellular zinc, basal FluoZin-3 fluorescence $(F)$ recorded from the slice increased slowly, although the stimulus-evoked $\Delta F$ remained stable.
Therefore, $\Delta F$ was measured to represent amount of transmitter release and $\Delta F$ had to be stable for at least $20 \mathrm{~min}$ before a manipulation was made. The short-term bleaching course of FluoZin-3 was corrected by linear extrapolation (see Fig. 1) instead of measuring actual dye bleaching time course as shown in our previous study (Qian and Noebels, 2005). Although this method of correction for bleaching is not perfect, it did not significantly affect the measurement of each individual $\Delta F$. More importantly, it reduced the amount of light exposure by $50 \%$, and ensured a stable $\Delta F$ over the $1.5 \mathrm{~h}$ period that is critical for LTD experiments. The fluorescence intensity in all figures was plotted in the arbitrary unit of photodiode output. Data in each experiment were normalized to the baseline before drug application, and then pooled and expressed as a mean \pm SD.

Pharmacological reagents. The zinc indicator FluoZin-3 was obtained from Invitrogen (San Diego, CA). DHPG, (2S)-2-amino-2-[(1S,2S)-2carboxycycloprop-1-yl]-3(xanth-9-yl)propanoic acid (LY341495), CNQX, and D-APV were purchased from Tocris (Ellisville, MO). Adenosine and baclofen were from RBI (Natick, MA). Neuropeptide Y (NPY ${ }_{3-36}$, rat) and $\omega$-conotoxin GVIA ( $\omega$-CgTX GVIA) were from Bachem (Torrance, CA). All other chemical reagents were obtained from Sigma (St. Louis, MO).

\section{Results \\ Detection of evoked vesicular zinc release by individual action potentials in the stratum radiatum of the hippocampal CA1 region \\ Figure $1 A$ is a schematic diagram detailing the measurement of} extracellular zinc accumulation $\left([\mathrm{Zn}]_{\mathrm{o}}\right)$ evoked by stimulating the CA3-CA1 synapse in the stratum radiatum of the hippocampal CA1 region of mouse brain slices. The top inset (Fig. 1 $\mathrm{Aa}$ ) shows the fluorescence response of the high affinity zinc indicator FluoZin-3 $\left([\mathrm{Zn}]_{\mathrm{t}}\right)$ evoked by a stimulation protocol consisting of five pulses at $5 \mathrm{~Hz}$. Over the sampling period of $2.5 \mathrm{~s}, \sim 1 \%$ of the signal diminished because of photobleaching of the zinc indicator. To obtain a more accurate time course of the $[\mathrm{Zn}]_{\mathrm{t}}$ decay, dye bleaching was corrected by linear extrapolation of the baseline before stimulation as shown by the dashed line. The bottom inset (Fig. 1 $A b$ ) shows sample traces of autofluorescence, autofluorescence in the presence of CNQX/D-APV, and the fluorescence response of FluoZin-3 after correction for photobleaching. Because there was a significant amount of postsynaptic activitydependent change of autofluorescence in the CA1 region, the glutamate receptor antagonists CNQX $(10 \mu \mathrm{M})$ and D-APV $(25$ $\mu \mathrm{M})$ were routinely added to the perfusate to eliminate the interference of autofluorescence in all zinc measurements. Figure $1 B$ summarizes the experimental results from wild-type $(+/+)$ and vesicular zinc transporter $Z n t 3$-deficient mutant mice $(Z n t 3-/-)$. As demonstrated here, CNQX and D-APV abolished the autofluorescence transient but not the $[\mathrm{Zn}]_{\mathrm{t}}$. The average peak amplitude of the $[\mathrm{Zn}]_{\mathrm{t}}$ was $2.1 \pm 0.5 \%(\Delta F / F)$ in wild-type mice $(n=24)$. However, no $[\mathrm{Zn}]_{\mathrm{t}}$ was observed in slices from $Z n t 3-/-$ mice $(n=5)$. These data confirm that the $[\mathrm{Zn}]_{\mathrm{t}}$ is a result of the interaction between vesicular zinc and FluoZin-3 after action potential-induced exocytosis of synaptic vesicles. The absence of an evoked FluoZin-3 signal in Znt3-/- mice also excludes the possibility of irrelevant artifacts such as light scattering contributing to the FluoZin-3 signal.

\section{Zinc release and glutamate release share the same short-term} facilitation and $\mathrm{Ca}^{2+}$ channel reliance at the CA3-CA1 synapse

Vesicular zinc coreleased with glutamate has been demonstrated to serve as a surrogate marker for presynaptic neurotransmitter release at the intensely zinc-rich hippocampal mossy fiber synapse (Qian and Noebels, 2005). In contrast to the mossy fiber synapse, where zinc coexists with glutamate in synaptic vesicles 
A
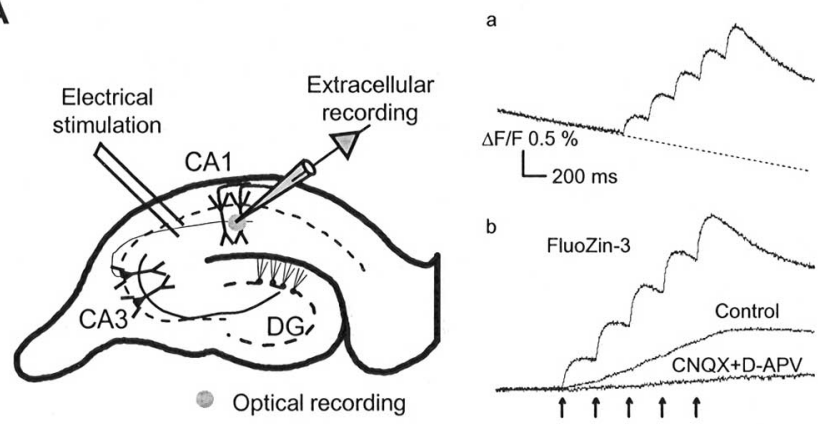

B
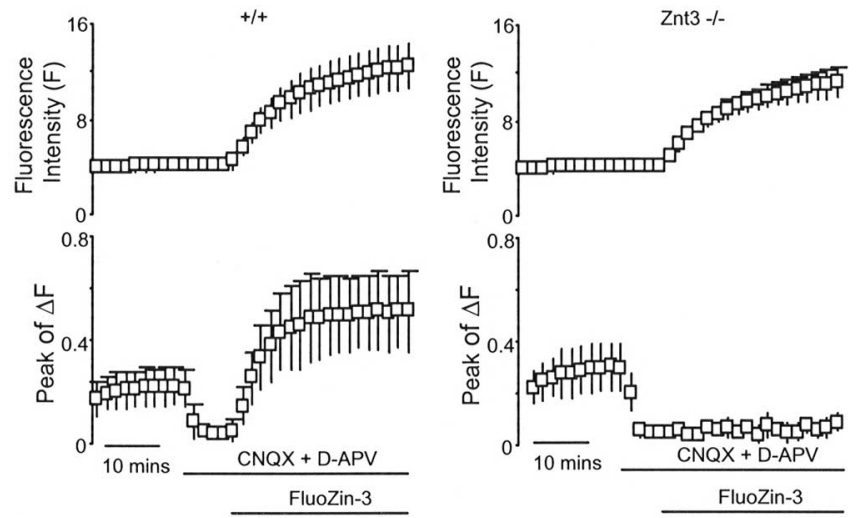

Figure 1. Vesicular zinc release evoked by individual action potentials at the CA3-CA1 synapse. $A$, Schematic diagram of a hippocampal slice showing stimulating and recording sites. DG, Dentate gyrus. Inset, Sample traces of autofluorescence and fluorescence signal of FluZin-3 in response to $5 \mathrm{~Hz}$ stimulation. $\boldsymbol{a}$, FluoZin-3 fluorescence trace before correction for photobleaching. $\boldsymbol{b}$, Autofluorescence in the presence of the glutamate receptor antagonist CNQX/D-APV and FluoZin-3 fluorescence after correction of photobleaching. The time course of the photobleaching was corrected by linear extrapolation of the time course before stimulation. $\boldsymbol{B}$, Left, Time course of the fluorescence intensity $(F)$ and the peak amplitude of the transient fluorescence change $\left(\Delta F\right.$ or $\left.[\mathrm{Zn}]_{\mathrm{t}}\right)$ in response to application of $C \mathrm{NQX} / \mathrm{D}-\mathrm{APV}$ and FluoZin-3 in wild-type mice $(n=24)$. Blockade of postsynaptic depolarization with CNQX/D-APV eliminated the autofluorescence transient but not the FluoZin-3 signal. Right, Time course of the fluorescence intensity $F$ and the peak amplitude of $\Delta F$ in response to application of CNQX/D-APV and FluoZin-3 in vesicular zinc transporter-deficient mice (Znt3-I-;n=5). Lack of FluoZin-3 signal in $\mathrm{Znt} 3-/-$ mice indicates that $[\mathrm{Zn}]_{\mathrm{t}}$ resulted from the interaction between vesicular zinc and FluoZin-3 after exocytosis of synaptic vesicles. Error bars present $S D$ in all figures.

within every mossy fiber bouton, only $\sim 45 \%$ of the boutons making axospinous glutamatergic synaptic contacts in the stratum radiatum of the CA1 region are zinc containing (Sindreu et al., 2003). We reasoned, however, that if zinc-positive synapses possess similar release properties as zinc-negative synapses in this pathway (see below), then $[\mathrm{Zn}]_{\mathrm{t}}$ measured at the zinc-positive synapses could be used as surrogate marker to represent neurotransmitter release at all CA3-CA1 synapses. Therefore, in the following experiments, we characterized zinc release at the zincpositive synapse and compared it with fEPSPs obtained from the entire CA3-CA1 population.

Figure $2 \mathrm{~A}$ summarizes the short-term facilitation of zinc release and of glutamate release. In contrast to a robust short-term facilitation of the $[\mathrm{Zn}]_{\mathrm{t}}$ at mossy fiber synapses (Qian and Noebels, 2005), the CA3-CA1 synapses here exhibited only moderate short-term facilitation, and the pattern of short-term facilitation of the $[\mathrm{Zn}]_{\mathrm{t}}$ was essentially similar to that measured for the fEPSP. As summarized in Figure $2 A$, the amplitudes of the $[\mathrm{Zn}]_{\mathrm{t}}$ corresponding to the first through fifth action potentials were $72 \pm 5,97 \pm 3,104 \pm 2,111 \pm 3$, and $116 \pm 4 \%$ of the mean value
A

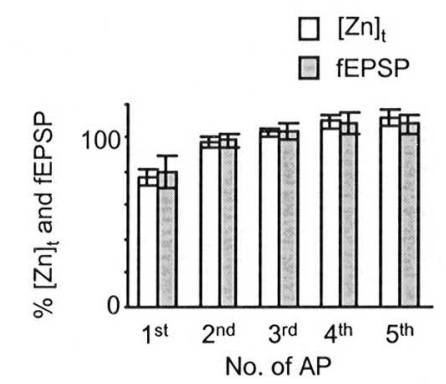

B
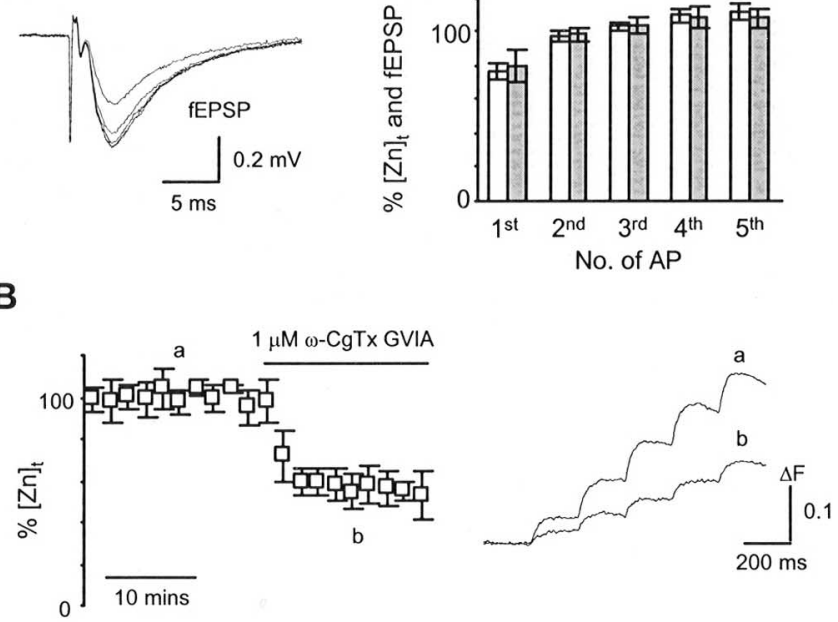

C
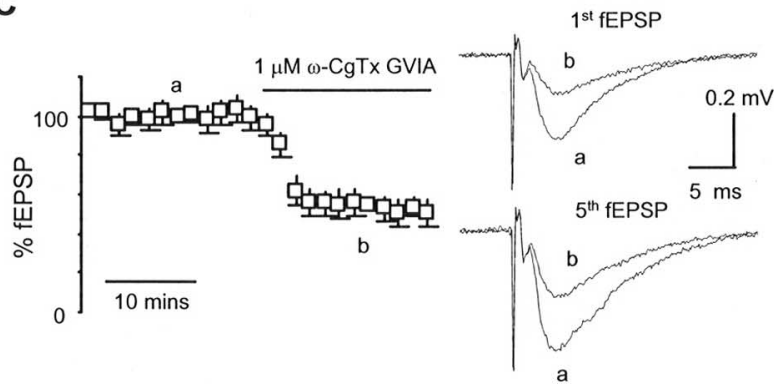

Figure 2. Short-term facilitation and $\mathrm{Ca}^{2+}$ channel reliance of zinc release are similar to glutamate release. $A$, Left, Superimposed fEPSPs evoked by the $5 \mathrm{~Hz}$ stimulation protocol. Right, Summary data of short-term facilitation of zinc release and glutamate release. Zinc release exhibits the same pattern of short-term facilitation as glutamate release. To quantify $\Delta F$ for each action potential, 10 traces of the $[\mathrm{Zn}]_{\mathrm{t}}$ during steady state were first averaged and then smoothed with an average time window of $10 \mathrm{~ms}$, and then the $\Delta F$ corresponding to each action potential (AP) was measured. The smoothing algorithm does not alter the kinetics of the $[\mathrm{Zn}]_{t}$. $B, C$, The N-type $\mathrm{Ca}^{2+}$ channel blocker $\omega$-CgTX GVIA equally inhibited the $[\mathrm{Zn}]_{\mathrm{t}}$ and fEPSP. The time course of the $[\mathrm{Zn}]_{\mathrm{t}}$ was quantified by measuring the total $\Delta$ F evoked by a $5 \mathrm{~Hz}$ stimulation protocol. To be better compared with the time course of the $[\mathrm{Zn}]_{t^{\prime}}$ a summation of all five fEPSPs within the stimulation train was quantified as the time course of fEPSP. Insets show sample traces of the $[\mathrm{Zn}]_{\mathrm{t}}$ (smoothed), first and fifth fEPSP before and after application of $\omega$-CgTx GVIA.

of all five $[\mathrm{Zn}]_{\mathrm{t}}(n=26)$, respectively. The fEPSPs corresponding to the first through fifth action potentials were $74 \pm 9,99 \pm 5$, $105 \pm 5,111 \pm 7$, and $110 \pm 6 \%$ of the average of all five fEPSPs $(n=27)$, respectively.

To determine the $\mathrm{Ca}^{2+}$ channel subtype reliance of zinc release at these synapses, we then examined the effect of the N-type $\mathrm{Ca}^{2+}$ channel blocker $\omega$-CgTx GVIA on the $[\mathrm{Zn}]_{\mathrm{t}}$. Figure $2 B$ shows the grouped time course of the $[\mathrm{Zn}]_{t}$ in response to application of $1 \mu \mathrm{m} \omega$-CgTx GVIA. The N-type $\mathrm{Ca}^{2+}$ channel blocker partially reduced the $[\mathrm{Zn}]_{\mathrm{t}}$ as shown by the time course of the $[\mathrm{Zn}]_{\mathrm{t}}$ and the sample traces taken during steady state. Figure $2 \mathrm{C}$ shows typical results of $\omega$-CgTX GVIA on the fEPSP at the CA3CA1 synapses. Blockade of $\mathrm{N}$-type channels resulted in an equal reduction of both the $[\mathrm{Zn}]_{\mathrm{t}}$ and fEPSP. On average, the $\Delta F \mathrm{~s}$ corresponding to the first and fifth action potentials were reduced to $47 \pm 6$ and $48 \pm 5 \%$ of baseline $(n=5)$, respectively. The corresponding fEPSPs were $48 \pm 6$ and $53 \pm 7 \%$ of baseline $(n=5)$. We did not examine the contribution of $\mathrm{P} / \mathrm{Q}$-type $\mathrm{Ca}^{2+}$ channels on the $[\mathrm{Zn}]_{\mathrm{t}}$, because blockade of P/Q-type $\mathrm{Ca}^{2+}$ channels, which 
contribute predominantly to the remainder of the presynaptic $\mathrm{Ca}^{2+}$ required for exocytosis at this synapse (Wheeler et al., 1994), should yield a similar result on the $[\mathrm{Zn}]_{\mathrm{t}}$ and fEPSP (see Discussion).

\section{Zinc release and glutamate release are modulated identically at CA3-CA1 synapses}

Next, we characterized the modulation of zinc release and compared it with that measured for the fEPSP. Neurotransmitter release at CA3-CA1 synapses is regulated by presynaptic receptors for NPY, $G_{A B A}$, and adenosine acting via presynaptic $\mathrm{Ca}^{2+}$ channels (Qian et al., 1997; Wu and Saggau, 1997). Figure $3, A$ and $B$, shows the effect of NPY on the $[\mathrm{Zn}]_{t}$ and fEPSP. Application of $1 \mu \mathrm{M}$ NPY led to a similar effect on both $[\mathrm{Zn}]_{t}$ and fEPSP. NPY inhibited the $[\mathrm{Zn}]_{\mathrm{t}}$ by $78 \pm 1$ and $79 \pm 1 \%(n=$ 4) for the first and fifth action potentials, respectively. The average reduction of the corresponding fEPSPs was $80 \pm 4$ and $78 \pm 1 \%(n=4)$. Similarly, activation of presynaptic $\mathrm{GABA}_{\mathrm{B}}$ and adenosine receptors resulted in the same amount of inhibition of both the $[\mathrm{Zn}]_{\mathrm{t}}$ and fEPSP. As shown in Figure 3, $C$ and $D$, baclofen (10 $\mu \mathrm{M})$, the $\mathrm{GABA}_{\mathrm{B}}$ receptor agonist, reduced the $[\mathrm{Zn}]_{\mathrm{t}}$ by $71 \pm 4$ and $70 \pm 5 \%(n=4)$ for the first and fifth action potentials, respectively. The mean reduction of the corresponding fEPSPs was $76 \pm 4$ and $68 \pm$ $4 \%(n=4)$. Not surprisingly, application of $5 \mu \mathrm{M}$ adenosine also reversibly inhibited the $[\mathrm{Zn}]_{\mathrm{t}}$ and fEPSPs to a similar extent (Fig. $3 E, F)$. The $[\mathrm{Zn}]_{\mathrm{t}}$ during the peak effect of adenosine was $54 \pm 11$ and $51 \pm 8 \%$ $(n=4)$ of baseline for the first and fifth action potentials, respectively, and the corresponding fEPSPs were $50 \pm 7$ and $53 \pm 8 \%(n=4)$ of baseline. These results, combined with similar short-term facilitation and $\mathrm{Ca}^{2+}$ channel reliance of neurotransmitter release, indicate that the same release machinery and regulatory mechanisms are operative at both zinc-positive and zinc-negative synapses. Therefore, $[\mathrm{Zn}]_{\mathrm{t}}$ at zinc-positive synapses in the CAl region is a valid surrogate marker to directly access global neurotransmitter release at the CA3-CA1 synapse.

\section{Persistent depression of neurotransmitter release after activation of mGluR}

Using fluorescence detection of zinc release, we then explored the expression site of mGluR-LTD with the mGluR agonist DHPG at this synapse. To compare results with previous studies, a test protocol consisting of paired pulses with an interval of $50 \mathrm{~ms}$ was first used to test DHPG-LTD at the CA3-CA1 synapse. In agreement with previous reports, a $10 \mathrm{~min}$ application of $100 \mu \mathrm{M}$ DHPG led to persistent depression of synaptic transmission at the CA3-CA1 synapse $(n=5)$ as shown in Figure $4 A$. The action of DHPG was completely blocked with the broad mGluR antagonist LY341495 (20 $\mu \mathrm{M} ; n=3$ ), indicating the requirement for mGluR activation in DHPG-LTD. DHPG-LTD was independent

\section{C}

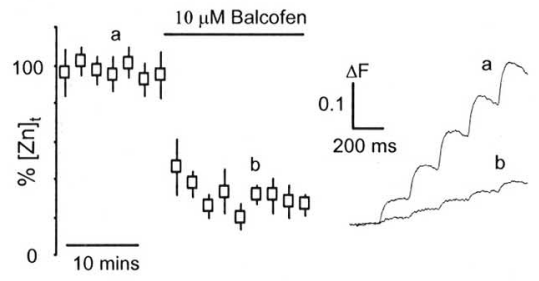

D

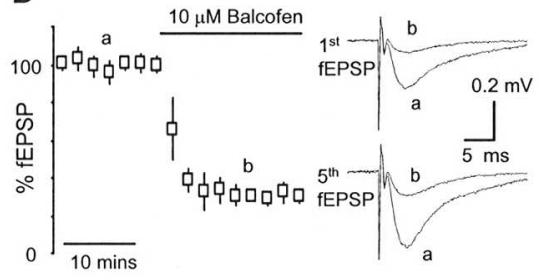

G

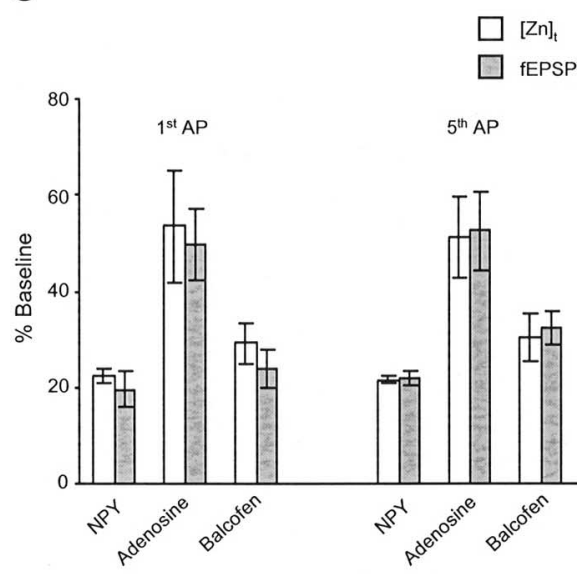

Figure 3. Identical modulation of zinc release and glutamate release. $\boldsymbol{A}-\boldsymbol{F}$, Modulation of zinc and glutamate release by NPY, described in Figure 2. Insets show sample traces of the [Zn $]_{t}$ (smoothed) and fEPSP taken at time points as marked. G, Summary data for the effects of NPY, baclofen, and adenosine on the $[\mathrm{Zn}]_{t}$ and fEPSP. The identical patterns of modulation, short-term facilitation, and $\mathrm{Ca}^{2+}$ channel reliance of zinc release and glutamate release confirm that the $[\mathrm{Zn}]_{t}$ is a valid surrogate marker for presynaptic neurotransmitter release at the CA3-CA1 synapse. AP, Action potential.

of the protocols used for testing. As shown in Figure $4 B$, a short application of DHPG in slices receiving the same stimulation protocol used in optical recordings induced an equivalent amount of LTD. D-APV $(25 \mu \mathrm{M})$ was routinely applied in this set of LTD experiments to eliminate a possible contribution from NMDA receptor-medicated LTD. The average fEPSP $40 \mathrm{~min}$ after DHPG application was $67 \pm 11$ and $72 \pm 9 \%$ of baseline for first and fifth fEPSP, respectively $(n=10)$.

We then examined presynaptic neurotransmitter release during DHPG-LTD by measuring the $[\mathrm{Zn}]_{\mathrm{t}}$ in response to DHPG. As shown in Figure $4 C$, after an initial decrease, the $[\mathrm{Zn}]_{\mathrm{t}}$ only partially recovered $40 \mathrm{~min}$ after washout of DHPG and exhibited a pattern of long-term depression. The first and fifth $[\mathrm{Zn}]_{\mathrm{t}}$ were $70 \pm 7$ and $69 \pm 7 \%$ of baseline, respectively $(n=5)$. The persistent reduction of the $[\mathrm{Zn}]_{\mathrm{t}}$ was not attributable to a possible deletion of vesicular zinc, because the $[\mathrm{Zn}]_{t}$ in control experiments remained at a stable level throughout the entire measurement. The first and fifth $[\mathrm{Zn}]_{\mathrm{t}}$ were $97 \pm 2$ and $95 \pm 3 \%(n=4)$ of baseline by the end of the experiments. Our results here clearly indicate a presynaptic expression of LTD after activation of mGluR at the CA3-CA1 synapse.

Recently, a developmental switch in the mechanism of 
A

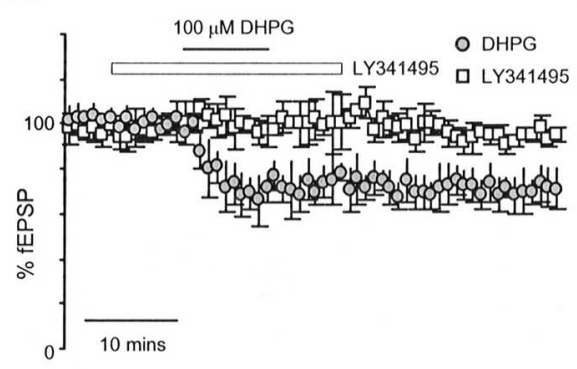

B

C

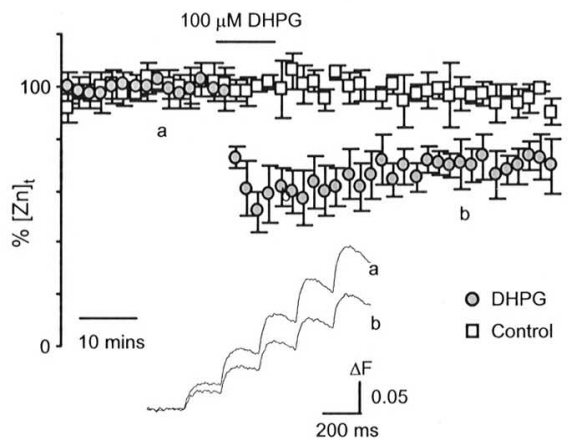

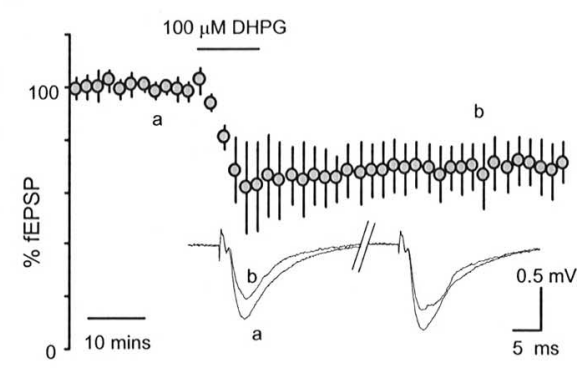

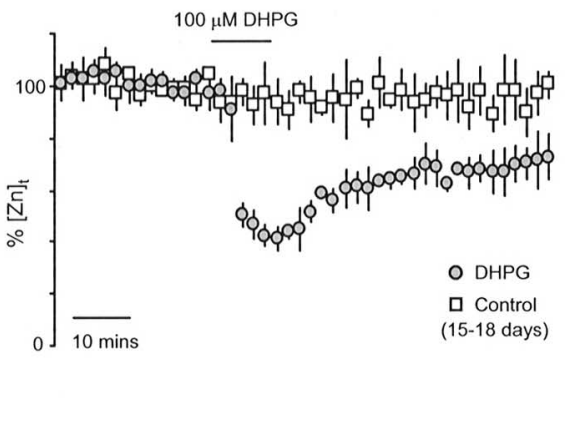

Figure 4. Persistent depression of neurotransmitter release after mGluR activation. $\boldsymbol{A}$, Time course of fEPSPs evoked by a pair of stimuli separated by an interval of $50 \mathrm{~ms}$ after a $10 \mathrm{~min}$ application of $100 \mu \mathrm{m}$ DHPG. The brief application of DHPG led to a persistent depression of synaptic transmission $(n=5)$ that was blocked by the nonspecific mGluR antagonist LY341495 $(n=3)$. $\boldsymbol{B}$, Time course of summation of fEPSPs evoked by $5 \mathrm{~Hz}$ stimulation protocol in response to the brief application of DHPG. The first and fifth fEPSPs were $67 \pm 11$ and $72 \pm 9 \%(n=10)$ of baseline and were obtained 40 min after washout of the DHPG.C, Time course of the $[\mathrm{Zn}]_{\mathrm{t}}$ in response to DHPG in adult mouse hippocampus (6-10 weeks). After DHPG application, there was a persistent depression of the $[\mathrm{Zn}]_{\text {. }}$. The first and fifth $\Delta F$ values were $70 \pm 7$ and $69 \pm 7 \%(n=5)$ of baseline when measured 40 min after washout of DHPG. The diminished $[\mathrm{Zn}]_{t}$ was not attributable to depletion of vesicular zinc, because the $[\mathrm{Zn}]_{t}$ remained at a stable level during the entire experimental period in the control group $(n=4)$. The persistent depression of the $[\mathrm{Zn}]_{\mathrm{t}}$ clearly indicates a presynaptic expression of DHPG-LTD. D, Time course of the $[\mathrm{Zn}]_{\mathrm{t}}$ in response to DHPG in developing brain (15-18 d). In slices from immature hippocampus, DHPG induced a greater initial depression of neurotransmitter release than in the adult group (C). However, the persistent depression of neurotransmitter release during DHPG-LTD was nearly identical in the two age groups. The first and fifth $\Delta F$ values were $67 \pm 7$ and $66 \pm 3 \%(n=5)$ of baseline when measured 40 min after washout of DHPG.

mGluR-LTD expression has been proposed, based on evidence that mGluR-LTD at neonatal synapses is mediated by presynaptic mechanisms, whereas mGluR-LTD at mature synapses may arise from postsynaptic modifications (Nosyreva and Huber, 2005). To learn whether presynaptic mechanisms of DHPG-LTD fade during brain maturation, we examined DHPG-LTD in developing mouse hippocampus. Figure $4 D$ summarizes the results from a group of young mice aged between 15 and $18 \mathrm{~d}$. When compared with the adult group (Fig. 4C), DHPG induced a greater initial depression of neurotransmitter release in immature slices. However, the amplitude of the later phase of DHPG-LTD was similar between the two age groups. On average, the first and fifth $[\mathrm{Zn}]_{\mathrm{t}}$ responses measured $40 \mathrm{~min}$ after DHPG application were $67 \pm 7$ and $66 \pm 3 \%$ of baseline, respectively $(n=5)$. The corresponding $[\mathrm{Zn}]_{\mathrm{t}}$ responses in the same age control group were $93 \pm 6$ and $95 \pm 7 \%$ of baseline, respectively $(n=5)$. These results indicate that the same presynaptic mechanism controls expression of DHPG-LTD in both developing and mature mouse brain.

\section{Discussion}

The ability to detect excytosis at zinc-containing synapses provides a novel method to analyze plasticity of the release process in hippocampal slices. Refinements in the method allow exploration of release dynamics in non-mossy fiber terminals, and here we have used the zinc fluorescence detection technique to explore the site of persistent depression of synaptic transmission at CA3-CA1 synapses after activation of mGluR with DHPG. Our results are congruent with previous conclusions based on electrophysiological and vesicle dye loading methods of the presynaptic site for LTD expression at CA3-CA1 synapses, and reveal additional properties of this heterogeneous presynaptic terminal population.

\section{Release properties of zinc-containing synapses at the CA3-CA1 synapses} In contrast to the mossy fiber pathway, where zinc coexists with glutamate in synaptic vesicles within every mossy fiber bouton, only about one-half of the CA3CA1 synapses in the stratum radiatum are zinc/Znt3 positive (Sindreu et al., 2003). This raises several key questions. First, where are the parent neurons of zinc containing synapses in this region? The vesicular zinc transporter gene $Z n t 3$ is strongly expressed in CA3 pyramidal neurons (Palmiter et al., 1996), consistent with its presence in fiber terminals in the Schaffer collateral pathway. The origin of zincnegative synapses, however, is less clear, and these terminals may represent a functional subgroup of neurons residing in CA3 or elsewhere (Sindreu et al., 2003). Second, do the zinc-positive synapses in this lamina possess different release properties and plasticity compared with zincnegative synapses? Using only conventional electrophysiological recording techniques, it is impossible to separate the zinc-positive synaptic component of the fEPSP from the one that is zinc negative; however, fluorescence detection of zinc release provides a direct way to selectively assess exocytosis at the zincpositive subpopulation. In this study, we find that neurotransmitter release at zinc-containing synapses relies on the same presynaptic $\mathrm{Ca}^{2+}$ channel subtype ratio as zinc-negative synapses. As shown in Figure 2, inhibition of the $[\mathrm{Zn}]_{t}$ by the N-type $\mathrm{Ca}^{2+}$ blocker $\omega$-CgTx GVIA was identical to that measured electrophysiologically for the global fEPSP, implying a similar degree of $\mathrm{N}$-type channel contribution to neurotransmitter release at both zinc-positive and zinc-negative synapses. In addition, presynaptic N- and P/Q-type $\mathrm{Ca}^{2+}$ channels are sensitive to various neuromodulators (Qian et al., 1997; Wu and Saggau, 1997). As shown in Figure 3, modulation of the $[\mathrm{Zn}]_{\mathrm{t}}$ at zinc-positive synapses by NPY, baclofen, and adenosine was indistinguishable from that measured electrophysiologically from the whole population (fEPSP). Because N-type $\mathrm{Ca}^{2+}$ channels are equally involved in release at both synapses, the similarity in modulation profile also implies that the contribution of $\mathrm{P} / \mathrm{Q}$-type $\mathrm{Ca}^{2+}$ channels to neurotransmitter release at the zinc-positive synapses is equivalent to that at zinc-negative synapses. Moreover, similar short-term facilitation of the $[\mathrm{Zn}]_{\mathrm{t}}$ and fEPSP (Fig. $2 \mathrm{~A}$ ) is consistent with the involvement of the same sets of presynaptic $\mathrm{Ca}^{2+}$ channels in the release process at both types of synapses. To- 
gether, our experimental results indicate that similar mechanisms control neurotransmitter release at both zinc-positive and zinc-negative glutamatergic synapses in the Schaffer collateral/ commissural pathway.

\section{Presynaptic expression of DHPG-LTD at the mouse CA3-CA1 synapse}

Contrasting results have been obtained in previous studies for the expression site of mGluR-LTD at the CA3-CA1 synapse. The presynaptic expression of $\mathrm{mGluR}$-LTD is supported by evidence of altered PPF or decreased mEPSC frequency during synaptically induced mGluR-LTD or DHPG-LTD (Oliet et al., 1997; Fitzjohn et al., 2001; Faas et al., 2002; Rouach and Nicoll, 2003; Nosyreva and Huber, 2005). Although an increase in the PPF or a decrease in mEPSC frequency has been taken as an indication of reduced neurotransmitter release, the internalization of postsynaptic AMPA receptors could also lead to a similar apparent change in the PPF or mEPSC frequency. The reverse process has been found in LTP (Poncer and Malinow, 2001), and indeed, the internalization or redistribution of AMPA receptors after application of DHPG has been reported in cultured hippocampal neurons (Snyder et al., 2001; Xiao et al., 2001). In slice preparations, mixed results have been obtained. Initially, DHPG was found to reduce synaptic transmission without altering the postsynaptic membrane sensitivity of glutamate in hippocampal CA1 neurons during DHPG-LTD, favoring a presynaptic mechanism (Rammes et al., 2003). Recently, however, a quantitative study of AMPA receptor expression in hippocampal slices revealed a reduction of AMPA receptor surface expression after application of DHPG in adolescent rats (but not immature) brains (Nosyreva and Huber, 2005). Additional evidence has been obtained with another surrogate marker for release, the membrane-labeling dye FM 1-43, which was used to label synaptic vesicle membrane during the cycling of presynaptic vesicles to allow comparison of the extent of labeling before and after LTD induction (Zakharenko et al., 2002). They found a decrease in vesicle cycling after LTD induction, which was the first result that did not rely on postsynaptic membrane response properties to support the evidence for the presynaptic expression of mGluR-LTD. However, there is an inherent sampling limitation linked with this method. In that study, a single evaluation of the vesicle cycling process lasts $20 \mathrm{~min}$ and consists of $2 \mathrm{~min}, 10 \mathrm{~Hz}$ burst stimulation for dye staining and $4.5 \mathrm{~min}, 1.5 \mathrm{~Hz}$ stimulation for dye destaining. Therefore, the time course of changes in vesicle cycling after induction could not be repetitively quantified, and the release profile was based on a single assay. In contrast, the zinc fluorescence imaging technique used in our study allows constant monitoring of neurotransmitter release throughout the entire experimental time period. The persistent reduction of zinc release after application of DHPG we observed provides new and convincing evidence for a presynaptic expression of DHPG-LTD. Nevertheless, our results do not rule out a parallel postsynaptic mechanism that may also occur at release-silent synapses brought on by DHPG.

\section{Mechanism of presynaptic depression}

A general agreement has been reached that mGluR-LTD is initially induced postsynaptically (Bolshakov and Siegelbaum, 1994; Oliet et al., 1997; Watabe et al., 2002), thereby suggesting a retrograde signal mediating the presynaptic expression of mGluRLTD. The molecule responsible for signaling a presynaptic change in release probability during the late phase of DHPG-LTD is still unidentified. Given the fact that postsynaptic AMPA and NMDA receptors were blocked with CNQX and D-APV to elim- inate the interference of autofluorescence in our zinc measurement, the generation of such a retrograde messenger must be independent of a postsynaptic $\mathrm{Ca}^{2+}$ triggered signal contributed via $\mathrm{Ca}^{2+}$ entry through either NMDA receptors or voltage-gated $\mathrm{Ca}^{2+}$ channels. In the Fitzjohn et al. (2001) study, DHPG was shown to induce LTD in the absence of extracellular $\mathrm{Ca}^{2+}$. This result implies that postsynaptic $\mathrm{Ca}^{2+}$ entry via NMDA or voltage-dependent calcium channels is not required for DHPGLTD, consistent with our conclusion. Because postsynaptic $\mathrm{Ca}^{2+}$ entry is required for synaptically induced mGluR-LTD (Bolshakov and Siegelbaum, 1994; Oliet et al., 1997; Otani and Connor, 1998), our result raises the possibility that DHPG-LTD may have a different mechanism. Because of the significant autofluorescence, it is not currently possible to precisely monitor zinc released by action potentials without blocking postsynaptic activity, and we were therefore unable to verify the presynaptic expression of mGluR-LTD induced solely by synaptic activity. Furthermore, as shown in Figure 4, $C$ and $D$, we found that the persistent depression of neurotransmitter release after application of DHPG was nearly identical in developing and mature mouse brain. Therefore, we were unable to confirm a developmental switch of the mGluR-LTD mechanism as reported in adolescent rats (Nosyreva and Huber, 2005). It is unclear whether the discrepancy between these two studies might be attributable to the species difference of experimental animals.

\section{Vesicular zinc exocytosis at non-mossy fiber terminals}

Zinc, as an essential divalent metal in the brain, exists in various forms throughout many subcellular compartments of the CNS (Frederickson et al., 2005). Previous studies of vesicular zinc exocytosis at synapses have naturally focused on the zinc-rich mossy fiber pathway in the hippocampus (Thompson et al., 2000; Li et al., 2001; Ueno et al., 2002; Kay, 2003; Qian and Noebels, 2005). Little attention has been paid to neuronal circuits with weak histochemical vesicular zinc staining, although the widespread presence of mRNA for $Z n t 3$, the gene underlying zinc transport into synaptic vesicles suggests that many glutamate synapses other than the mossy fiber synapse may also have zinc available for release (Palmiter et al., 1996). Because of differences in dye concentration and stimulus strength required to resolve vesicular zinc exocytosis in the CA1 region, the amplitudes of $\Delta F / F$ are not directly comparable with those reported at mossy fiber synapses (Qian and Noebels, 2005). Our zinc signal neither reflects absolute levels of zinc, nor distinguishes between free zinc and the externalization of membrane-bound zinc in the synaptic cleft as discussed by Kay (2006). In summary, the enhanced ability to resolve exocytosis of vesicular zinc evoked by action potentials opens the exploration of presynaptic release dynamics at many other zinc-containing neuronal circuits in the CNS.

\section{References}

Bolshakov VY, Siegelbaum SA (1994) Postsynaptic induction and presynaptic expression of hippocampal long-term depression. Science 264:1148-1152.

Faas GC, Adwanikar H, Gereau RW, Saggau P (2002) Modulation of presynaptic calcium transients by metabotropic glutamate receptor activation: a differential role in acute depression of synaptic transmission and long-term depression. J Neurosci 22:6885-6890.

Fagni L, Chavis P, Ango F, Bockaert J (2000) Complex interactions between mGluRs, intracellular $\mathrm{Ca}^{2+}$ stores and ion channels in neurons. Trends Neurosci 23:80-88.

Fitzjohn SM, Palmer MJ, May JE, Neeson A, Morris SA, Collingridge GL (2001) A characterisation of long-term depression induced by metabotropic glutamate receptor activation in the rat hippocampus in vitro. J Physiol (Lond) 537:421-430. 
Frederickson CJ, Suh SW, Silva D, Frederickson CJ, Thompson RB (2000) Importance of zinc in the central nervous system: the zinc-containing neuron. J Nutr 130:1471S-1483S.

Frederickson CJ, Koh JY, Bush AI (2005) The neurobiology of zinc in health and disease. Nat Rev Neurosci 6:449-462.

Huang CC, Hsu KS (2006) Sustained activation of metabotropic glutamate receptor 5 and protein tyrosine phosphatases mediate the expression of (S)-3,5-dihydroxyphenylglycine-induced long-term depression in the hippocampal CA1 region. J Neurochem 96:179-194.

Kay AR (2003) Evidence for chelatable zinc in the extracellular space of the hippocampus, but little evidence for synaptic release of $\mathrm{Zn}$. J Neurosci 23:6847-6855.

Kay AR (2006) Imaging synaptic zinc: promises and perils. Trends Neurosci 29:200-206.

Li Y, Hough CJ, Suh SW, Sarvey JM, Frederickson CJ (2001) Rapid translocation of $\mathrm{Zn}^{2+}$ from presynaptic terminals into postsynaptic hippocampal neurons after physiological stimulation. J Neurophysiol 86:2597-2604.

Nosyreva ED, Huber KM (2005) Developmental switch in synaptic mechanisms of hippocampal metabotropic glutamate receptor-dependent longterm depression. J Neurosci 25:2992-3001.

Oliet SH, Malenka RC, Nicoll RA (1997) Two distinct forms of long-term depression coexist in CA1 hippocampal pyramidal cells. Neuron 18:969-982.

Otani S, Connor JA (1998) Requirement of rapid $\mathrm{Ca}^{2+}$ entry and synaptic activation of metabotropic glutamate receptors for the induction of longterm depression in adult rat hippocampus. J Physiol (Lond) 511:761-770.

Palmiter RD, Cole TB, Quaife CJ, Findley SD (1996) ZnT-3, a putative transporter of zinc into synaptic vesicles. Proc Natl Acad Sci USA 93:14934-14939.

Poncer JC, Malinow R (2001) Postsynaptic conversion of silent synapses during LTP affects synaptic gain and transmission dynamics. Nat Neurosci 4:989-996.

Qian J, Noebels JL (2005) Visualization of transmitter release with zinc fluorescence detection at the mouse hippocampal mossy fibre synapse. J Physiol (Lond) 566:747-758.

Qian J, Colmers WF, Saggau P (1997) Inhibition of synaptic transmission by neuropeptide $\mathrm{Y}$ in rat hippocampal area CA1: modulation of presynaptic $\mathrm{Ca}^{2+}$ entry. J Neurosci 17:8169-8177.

Rammes G, Palmer M, Eder M, Dodt HU, Zieglgansberger W, Collingridge GL (2003) Activation of mGlu receptors induces LTD without affecting postsynaptic sensitivity of CA1 neurons in rat hippocampal slices. J Physiol (Lond) 546:455-460.

Rouach N, Nicoll RA (2003) Endocannabinoids contribute to short-term but not long-term mGluR-induced depression in the hippocampus. Eur J Neurosci 18:1017-1020.

Sindreu CB, Varoqui H, Erickson JD, Perez-Clausell J (2003) Boutons containing vesicular zinc define a subpopulation of synapses with low AMPAR content in rat hippocampus. Cereb Cortex 13:823-829.

Snyder EM, Philpot BD, Huber KM, Dong X, Fallon JR, Bear MF (2001) Internalization of ionotropic glutamate receptors in response to $\mathrm{mGluR}$ activation. Nat Neurosci 4:1079-1085.

Thompson RB, Whetsell Jr WO, Maliwal BP, Fierke CA, Frederickson CJ (2000) Fluorescence microscopy of stimulated $\mathrm{Zn}$ (II) release from organotypic cultures of mammalian hippocampus using a carbonic anhydrase-based biosensor system. J Neurosci Methods 96:35-45.

Ueno S, Tsukamoto M, Hirano T, Kikuchi K, Yamada MK, Nishiyama N, Nagano T, Matsuki N, Ikegaya Y (2002) Mossy fiber $\mathrm{Zn}^{2+}$ spillover modulates heterosynaptic $N$-methyl-D-aspartate receptor activity in hippocampal CA3 circuits. J Cell Biol 158:215-220.

Watabe AM, Carlisle HJ, O’Dell TJ (2002) Postsynaptic induction and presynaptic expression of group $1 \mathrm{mGluR}$-dependent LTD in the hippocampal CA1 region. J Neurophysiol 87:1395-1403.

Wheeler DB, Randall A, Tsien RW (1994) Roles of N-type and Q-type Ca ${ }^{2+}$ channels in supporting hippocampal synaptic transmission. Science 264:107-111.

Wu LG, Saggau P (1997) Presynaptic inhibition of elicited neurotransmitter release. Trends Neurosci 20: 204-212.

Xiao MY, Zhou Q, Nicoll RA (2001) Metabotropic glutamate receptor activation causes a rapid redistribution of AMPA receptors. Neuropharmacology 41:664-671.

Zakharenko SS, Zablow L, Siegelbaum SA (2002) Altered presynaptic vesicle release and cycling during mGluR-dependent LTD. Neuron 35:10991110 . 